\title{
Sensoriamento Remoto e Estudos de Clima Urbano
}

\author{
Remote Sensing and Urban Climate Studies
}

\author{
Mariane Batista de Lima Moraes Brandão Campos*a; Victor Hugo de Morais Danelichen ${ }^{a}$
}

âniversidade de Cuiabá, Programa de Pós-Graduação Stricto Sensu em Ciências Ambientais. MT, Brasil.

*E-mail: mariane.campos@vgd.ifmt.edu.br

\begin{abstract}
Resumo
Em regiões onde a rede meteorológica não apresenta uma cobertura satisfatória o uso do sensoriamento remoto se apresenta como uma técnica eficaz para estudo ambiental, possibilitando examinar a métrica dos padrões de uso do solo, bem como análises espaciais e temporais do clima nas cidades. Dessa forma, este trabalho busca analisar a produção científica sobre as técnicas de predição de temperatura e umidade do ar a partir de dados de sensoriamento remoto. Foi realizada uma análise bibliográfica e documental em consultas à base de pesquisa da Scopus (Elseivier) com a seleção final de 15 artigos que possuem relação direta com o conteúdo da pesquisa e enfatizam o estudo de clima urbano pelos padrões de uso do solo urbano, analisando-se dados obtidos por meio de sensores orbitais de satélites como os da série Landsat. O resultado da pesquisa aponta para o uso da vegetação como importante recurso de mitigação dos efeitos negativos do clima na cidade. Modelos matemáticos estão sendo aprimorados para obtenção de temperatura do ar com base em dados de temperatura de superfície, uma vez que ambas variáveis possuem uma forte correlação. Os índices espectrais NDMI e NDWI são úteis na verificação de dados sobre umidade do ar, porém pouco consistentes em locais de vegetação nula ou esparsa. Identificou-se o sensoriamento remoto como ferramenta promissora inclusive na análise de mesoclimas e microclimas urbanos, sendo importante a continuidade de pesquisas e estudos que identifiquem suas potencialidades.
\end{abstract}

Palavras-chave: Umidade do Ar. Sensores Orbitais. Temperatura do Ar.

\begin{abstract}
In regions where the meteorological network does not have a satisfactory coverage or the use of remote sensing, it presents itself as an effective technique for environmental studies, allowing the metrics evaluation of land use patterns as well as spatial and temporal analyzes of the climate in cities. In this way, this research aims to analyze the scientific production on the temperature and humidity techniques prediction from remote sensing data. A bibliographic and documentary analysis was carried out in consultation with the Scopus (Elseivier) research base with a final selection of 15 scientific articles that directly relate to the research content and emphasize the study of urban climate by urban land use patterns, analyzing data obtained using satellite orbital sensors such as the Landsat series. The result of the research points to the use of vegetation as an important resource to mitigate the negative effects of climate in the city. Mathematical models are being improved to obtains air temperature based on surface temperature data, since both variables have a strong correlation. The NDMI and NDWI spectral indices are useful in verifying data on air humidity, but they are not very consistent in areas of null or sparse vegetation. Remote sensing has been identified as a promising tool, including in the analysis of urban mesoclimates and microclimates, and it is important to continue research and studies that identify its potential.
\end{abstract}

Keywords: Air Humidity. Orbital Sensors. Air Temperature.

\section{Introdução}

As transformações nas superfícies do solo oriundas dos processos de urbanização intensa e acelerada tem sido apontado como o principal causador de mudanças climáticas nas cidades (YU et al., 2017; NOVAIS et al., 2018; FERREIRA, 2019).

Nesse sentido, a substituição de superfícies vegetadas, em especial, por áreas impermeabilizadas - asfalto, concreto, cerâmica, dentro outros - no processo de formação das cidades, causam mudanças no equilíbrio do ambiente urbano, diminuindo a umidade provenientes dos processos de evapotranspiração, elevando as temperaturas do solo e consequentemente do ar, provocando alterações no clima das cidades que geram efeitos como as ilhas de calor urbana (CALLEJAS et al., 2011; ZHANG et al., 2018, YU et al., 2017; YANG et al., 2017a; DAI et al., 2018; MARANDO et al., 2019).

Dessa forma, temperatura do ar e umidade ar são, portanto, elementos chaves no entendimento do funcionamento do clima urbano uma vez que interferem diretamente na forma como sentimos o ambiente das cidades e também na qualidade de vida das pessoas, o que implica que estudos de umidade e temperatura do ar tem aplicações em diversas áreas, incluindo o planejamento urbano (FILGUEIRAS et al., 2016; NOVAIS et al., 2018).

Atualmente a maioria das pesquisas envolvendo clima urbano, seja em uma escala de mesoclima ou microclima, 
envolvendo variáveis de temperatura e umidade do ar são realizadas a partir de dados coletados por equipamentos específicos operados ao nível do solo, por meio de trasnsectos móveis e/ou dados coletados de estações meteorológicas (HENDEL et al., 2016; NOVAIS et al., 2017; YANG et al., 2017b; WANG et al., 2017; LIN et al., 2017; KOTHARK; BAGAGE, 2018; ZHOU; CHEN, 2018).

Filgueiras et al. (2016), Liu et al. (2016), Zhang et al. (2018), Souza et al. (2017) e Dai et al. (2018) ponderam que em locais onde a rede de estações meteorológicas é implantada em um número limitado de locais, e por isso muitas vezes as informações, que geralmente são estimadas por interpolação, não são precisas e não representam a variabilidade espacial de forma detalhada. Por sua vez, as medidas coletadas nos locais de estudo demandam equipamentos específicos, muitas vezes de alto custo e equipe especializada com disponibilidade para longas pesquisas de campo.

Diante disso, o sensoriamento remoto se apresenta como uma alternativa no estudo do clima para regiões que possuam escassez de informações meteorológicas, uma vez que representa uma técnica de baixo custo, fazendo uso de equipamentos não intrusivos e já programados para coleta sistematizada de dados (FILGUEIRAS et al., 2016; GAIDA et al., 2020). O presente estudo, portanto, busca analisar a produção cientifica sobre as técnicas de predição de temperatura e umidade do ar a partir de dados de sensoriamento remoto.

\section{Desenvolvimento}

\subsection{Metodologia}

O presente trabalho trata de uma revisão de estudos do clima por sensoriamento remoto realizado através de consultas à base de pesquisa da Scopus (Elseivier) disponível através do Portal de Periódicos da CAPES/MEC.

Para desenvolvimento desta revisão foram definidos alguns critérios de inclusão tais como: artigos publicados nos últimos cinco anos e disponíveis de forma completa, artigos relacionados com ciências ambientais e sem restrições de país ou território.

Sendo o objetivo da pesquisa estudar métodos de predição de temperatura e umidade do ar via sensoriamento remoto, buscou-se restringir a busca da bibliografia na base de pesquisa através da seguinte chave de procura: (umidade OU temperatura) E medição) E ("parque urbano").

Como resultado da busca de pesquisa conforme parâmetros especificados nos parágrafos anteriores, foram encontrados 140 artigos referentes a estudo de temperatura e umidade do ar, a grande maioria tratando sobre ilhas de calor. Do total de 140 artigos elencados, 32 artigos tratavam do estudo de temperatura e umidade do ar através do uso de sensoriamento remoto e desse afunilamento 15 artigos (Quadro 1) foram selecionados para desenvolvimento deste trabalho por possuírem relação direta com o conteúdo de estudo desta pesquisa.

Quadro 1 - Lista de artigos selecionados para pesquisa na base de dados Scopus 2016 - 2020

\begin{tabular}{|c|c|c|c|}
\hline Autores & Periódicos & Ano & Local \\
\hline PELTA, R. et al. & Environmental Pollution & 2016 & Israel \\
\hline LIU, S. et al. & Advances in Meteorology & 2016 & China \\
\hline SCHULTZ, M. et al. & International Journal of Applied Earth Observation and Geoinformation & 2016 & Holanda \\
\hline ESTOQUE, R. C. et al. & Science of the Total Environment & 2017 & Japão \\
\hline CHENG, K. et al. & Landscape and Urban Planning & 2017 & Taiwan \\
\hline YU, Z. et al. & Ecological Indicators & 2017 & China \\
\hline YANG, C. et al. & Sustainability (Switzerland) & 2017 & China \\
\hline PELTA, R. et al. & Science of The Total Environment & 2017 & Israel \\
\hline ZHANG, X. et al. & International Journal of Environmental Research and Public Health & 2018 & China \\
\hline YU, Z. et al. & Urban Forestry and Urban Greening & 2018 & China \\
\hline DAI, Z. et al. & Science of the Total Environment & 2018 & China \\
\hline INDRAWATI, L. et al. & IOP Conference Series: Earth and Environmental Science & 2019 & Indonésia \\
\hline MARANDO, F. et al. & Ecological Modelling & 2019 & Itália \\
\hline GUO, G. et al. & Science of the Total Environment & 2019 & China \\
\hline LEMUS-CANOVAS, M. et al. & Science of the Total Environment & 2020 & Espanha \\
\hline
\end{tabular}

Fonte: Dados da pesquisa.

\subsection{Superfícies impermeabilizadas e Superfícies vegetadas}

As diversas atividades antrópicas sobre o ambiente urbano ocasionam mudanças no padrão do uso do solo originando, dentre outras consequências, fenômenos climáticos como as ilhas de calor urbana (ICU) que tendem a agravar o estresse térmico, o aumento do consumo de energia e a poluição do ar nas cidades, gerando impactos negativos para saúde e conforto de seus habitantes (YU et al., 2017).
As consequências negativas das ICU são ocasionadas por processos de retirada ou substituição da superfície natural vegetada por superfícies impermeabilizadas compostas por materiais absorvedores de calor que provocam o aquecimento da cidade (ESTOQUE et al., 2017, DAI et al., 2018).

A variabilidade de material que compõe a malha urbana, portanto, denota que o aumento das temperaturas das superfícies e do ar podem variar segundo varia o padrão da 
paisagem que é determinada pelos diversos tipos de uso do solo. Nos estudos dos mosaicos das cidades e sua relação com as ICU (CHENG et al., 2017; ESTOQUE et al., 2017; YU et al., 2017; YANG et al., 2017a) afirmam que a proporção entre superfície impermeabilizada e os espaços verdes representa um caminho de mitigação dos problemas de clima urbano apontando a presença de áreas vegetadas como parques e florestas urbanas, áreas de preservação permanente e arborização de ruas, um importante serviço de ecossistema urbano.

Zhang et al. (2018), Yu et al. (2018) e Guo et al. (2019) acrescentam ainda que apenas a presença de vegetação no espaço urbano não é suficiente para uma diminuição eficiente dos efeitos das ICU, devendo-se ponderar sobre a abundância, o tipo, a métrica e a geometria das manchas verdes e a interseção e/ou dispersão dessas com as superfícies impermeabilizadas.

Sendo assim, a vegetação inserida com o devido planejamento dentro da paisagem urbana consegue absorver o calor pelos processos de evapotranspiração bem como absorve radiação de ondas curtas, funcionando como um termorregulador do microclima da cidade, mantendo os ciclos biogeoquímicos da natureza e colaborando efetivamente com o equilíbrio climático dentro das áreas urbanas (YU et al., 2017; ZHANG et al., 2018; GUO et al., 2019).

Cheng et al. (2017) e Estoque et al. (2017) consideram que na métrica da paisagem, conhecer o padrão de uso do solo em uma cidade e a distribuição espacial de superfícies impermeabilizadas e vegetadas é um conveniente instrumento para se entender os níveis de urbanização e reunir informações que sirvam de base para tomada de decisões nos processos de planejamento urbano.

\subsection{Sensoriamento remoto e estudo dos padrões de superfícies urbanas}

O sensoriamento remoto (SR), segundo com Cheng et al. (2017), Yang et al. (2017a) e Indrawati et al. (2019) possibilita uma análise espacial e temporal mais precisa dos padrões de uso do solo colaborando para o exame apurado de variação da métrica da paisagem urbana sendo considerado uma ferramenta útil no estudo de clima e planejamento urbanos.

Nas investigações sobre a variabilidade de superfícies da cidade o SR orbital possibilita registros de temperatura superficial para cada cobertura do solo permitindo se estabelecer a sua distribuição espacial (LEMUS-CANOVAS et al., 2020).

Seguindo essa mesma linha de observação, Pelta et al. (2016) assegura que a variabilidade espacial da temperatura superficial, medida por sensoriamento remoto orbital, é capaz de fornecer um panorama das características da superfície da cidade e a variabilidade temporal da temperatura superficial evidencia as mudanças do uso do solo urbano.

Compreender a estrutura da paisagem urbana e relacionála com as temperaturas de superfície da cidade possibilita a reunião de importantes informações para estudo e mitigação de problemas de conforto ambiental urbano como as ICU (DAI et al., 2017).

De todos os artigos examinados nessa pesquisa o uso dos satélites da série Landsat são os mais utilizados pois, de acordo com Zhang et al. (2018) e Yang et al. (2017a) possui a série mais longa de observação da superfície terrestre e por estar equipado com o imageador OLI que possui precisão radiométrica de 12 bits e o sensor TIRS permite uma melhor compreensão dos padrões de cobertura do solo sendo útil na medição dos valores de temperatura superficial, variável importante na identificação de distúrbios de conforto térmico.

\subsection{Temperatura de Superfície e Temperatura do ar}

A temperatura do ar é uma das principais variáveis buscadas e medidas para estudos de climatologia, sendo útil para indicação de mudanças climáticas e descrição de trocas de energia (LIU et al., 2016).

Diversos estudos, contudo, utilizam as medidas de temperatura de superfície, identificadas a partir de sensor infravermelho térmico de satélites orbitais, para avaliar ilhas de calor e de frescor urbano, clima urbano e entender a evolução e variabilidade do uso do solo da cidade (SCHULTZ et al., 2016; PELTA et al., 2016; LIU et al., 2016; ESTOQUE et al., 2017; CHENG et al., 2017; YU et al., 2017; YANG et al., 2017a; ZHANG et al., 2018; PELTA; CHUDNOVSKY, 2017; YU et al., 2018; DAI et al., 2018; INDRAWATI et al., 2019; MARANDO et al, 2019; GUO et al., 2019; LEMUSCANOVAS et al., 2020).

Para cada tipo de cobertura do solo temos variações diferentes de inércia térmica e albedo que influenciam diretamente na variação dos valores de temperatura superficial. Análises de temperatura da superfície urbanas demonstram uma forte correlação positiva desta com o solo impermeabilizado, e uma correlação negativa com o solo vegetado (ESTOQUE et al., 2017; CHENG et al., 2017; YANG et al., 2017a; DAI et al., 2018; YU et al., 2018; LEMUS-CANOVAS et al., 2020) indicando a temperatura da superfície como um parâmetro importante para estudarmos qualidade ambiental urbana, clima e planejamento urbano.

É prudente assinalarmos aqui que a temperatura do ar indica a temperatura da atmosfera e geralmente é medida à altura de aproximadamente 2 metros do solo por estações meteorológicas levando em consideração os vórtices horizontais. A temperatura de superfície, medida por sensoriamento remoto orbital, indica a temperatura do solo aquecido. Sendo assim, quando medimos temperatura superficial não estamos medindo a temperatura do ar, porém várias pesquisas (PELTA et al., 2016; LIU et al., 2016; ZHANG et al., 2018; YU et al., 2017; DAI et al., 2018; YU et al., 2018; MARANDO et al., 2019) indicam uma forte correlação da temperatura superficial recuperada de imagens de sensores infravermelhos termais com a temperatura do ar.

Liu et al. (2016) aponta o método de obtenção de 
temperatura do ar através da temperatura da superfície utilizando o índice temperatura da vegetação - TVX, baseado no NDVI, o qual assume que a temperatura do ar próximo a superfície se aproxima da temperatura do dossel, porém salienta que é limitado pois não traz resultados consistentes em locais que apresentam nenhuma ou vegetação esparsa.

Liu et al. (2016) ainda propõe estudos para se obter temperatura do ar através de dados de temperatura da superfície desenvolvendo um modelo que aprimora o modelo ADABAT - equilíbrio de energia de advecção para a temperatura do ar. O modelo ADABAT foi desenvolvido por Zhang et al. (2015) e considera que a temperatura do ar na superfície é composta pela temperatura local e a temperatura exótica considerando as difusões turbulentas do ar e a troca de advecção horizontal.

O modelo proposto por Liu et al. (2016) recebeu nome de IADABAT e acrescenta ao modelo ADABAT o fator de advecção " $\mathrm{f}$ " através do método de ponderação por distância inversa - IDW.

\subsection{Umidade do ar e o índice de umidade por diferença normalizada - NDMI}

A umidade do ar diz respeito a quantidade de vapor de água na atmosfera sendo a variável que caracteriza se o ar é seco ou úmido. Nas superfícies impermeabilizadas das cidades a ausência de efeitos evaporativos tendem a provocar o aumento da temperatura da superfície e do ar elevando as temperaturas médias urbanas (PELTA et al., 2016).

A umidade analisada através de dados de sensoriamento remota é feita através de índices de vegetação, como o índice de umidade por diferença normalizada - NDMI muito utilizado para mapeamento e monitoramento de vegetação (SCHULTZ et al., 2016; INDRAWATI et al., 2019), o índice de água por diferença normalizada modificado - MNDWI o índice de diferença normalizada da água - NDWI, sendo utilizados para identificar corpos de água e a espessura de água líquida da vegetação no espaço próximo ao dossel (GAO, 1996; ESTOQUE et al., 2017).

$\mathrm{O}$ índice de umidade por diferença normalizada (NDMI) é um índice espectral do Landsat que deriva da refletância da superfície e é usado para descrever o nível de estresse hídrico da vegetação através da determinação do teor de água dessa vegetação (USGS, 2017). Seu cálculo é feito através da razão entre a diferença e a soma das faixas de frequência refratadas no infravermelho próximo (NIR) e do Infravermelho próximo de ondas curtas (SWIR).

Os índices de diferença normalizada da água (NDWI) é um índice espectral relacionada a água líquida que deriva da reflectância da superfície e é usado para identificar e monitorar corpos de água e também monitorar as alterações no teor de água do dossel da vegetação. (GAO, 1996). Seu cálculo também é feito utilizando-se o infravermelho próximo (NIR) e o infravermelho próximo de ondas curtas (SWIR).

Os índices de vegetação NDMI e NDWI relacionados à umidade e água apresentam um bom desempenho para áreas densamente vegetadas, mas sua contribuição é negativa no estudo de superfícies parcialmente vegetadas, com nenhuma ou pouca vegetação. (GAO, 1996).

\section{Conclusão}

A partir dos estudos bibliográficos realizados constatou-se que o sensoriamento remoto (SR) é uma técnica bem aceita no estudo de mesoclimas e microclimas urbanos, sendo os dados oriundos dos satélites da série Landsat os mais comumente utilizados devido ao longo tempo de observação do planeta bem como por serem equipados com a tecnologia do Sensor Infravermelho Térmico - TIRS capaz de medir a temperatura da superfície terrestre.

Os dados medidos de temperatura do ar, de acordo com os autores pesquisados, apresentam uma forte correlação com dados de temperatura de superfície obtidos através de sensores orbitais do que se deduz que esta última representa um importante variável para estudo do clima na cidade e também é útil no estudo do entendimento e formação do mosaico urbano.

Analisando-se os estudos de umidade do ar via sensoriamento remoto é consoante o uso dos índices de vegetação NDWI e NDMI porém a consistência dos resultados está atrelada a presença de área vegetada sendo pouco eficientes para estudo de superfícies impermeabilizadas ou com pouca vegetação.

Alguns autores que pesquisam clima urbano através de técnicas de sensoriamento remoto propõem modelos matemáticos mais precisos para obtenção de dados de clima a partir de dados de satélite, contudo com resultados ainda pouco conclusivos e que necessitam de estudos mais numerosos e aprofundados.

\section{Referências}

CALLEJAS, I.J.A. et al. Uso do Solo e Temperatura Superficial em Área Urbana. Mercator, v. 10, n. 23, p. 207-223, 2011. doi: 10.4215/RM2011.1023.0015

CHENG, K. et al. Does urbanization increase diurnal land surface temperature variation? Evidence and implications. Landscape Urban Planning, v. 157, p. 247-258, 2017. doi:10.1016/j. landurbplan.2016.06.014

DAI, Z. et al. Spatial regression models of park and landuse impacts on the urban heat island in central Beijing. Scie. Total Environ., v.626, p.1136-1147, 2018. doi:10.1016/j. scitotenv.2018.01.165

ESTOQUE, R.C. et al. Effects of landscape composition and pattern on land surface temperature: An urban heat island study in the megacities of Southeast Asia. Scie.Total Environ., v.577, p.349-359, 2017. doi:10.1016/j.scitotenv.2016.10.195

FERREIRA, L. S. et al. Exploring the relationship between urban form, land surface temperature and vegetation indices in a subtropical megacity. Urban Climate, v.27, p. 105-123, 2019. doi: 10.1016/j.uclim.2018.11.002

FILGUEIRAS, R. et al. Predição da temperatura do ar por meio de sensoriamento remoto orbital. Rev. Bras. Cartog., n.68, p.1485-1495, 2016. 
GAIDA, W. et al. Correção atmosférica em sensoriamento remoto: uma revisão. Rev Bras. Geog. Fís., v.13, n.1, p.229, 2020. doi:10.26848/rbgf.v13.1.p229-248

GAO, B. NDWI A Normalized difference water index for remote sensing of vegetation liquid water from space. Remote Sensing Environ., v.58, n.3, p.257-266, 1996.

GUO, G. et al. Complex mechanisms linking land surface temperature to greenspace spatial patterns: evidence from four southeastern Chinese cities. Scie. Total Environ., v.674, p.77-87, 2019. doi:10.1016/j.scitotenv.2019.03.402

HENDEL, M. et al. Measuring the effects of urban heat island mitigation techniques in the field: Application to the case of pavement-watering in Paris. Urban Climate, v.16, p.43-58, 2016. doi:10.1016/j.uclim.2016.02.003

INDRAWATI, L. et al. Effect of Urban Expansion intensity on urban ecological status utilizing remote sensing and gis: a study of Semarang-Indonesia. IOP Conference Series: Earth and Environ.l Scie., v.451, n.1, 2019. doi:10.1088/1755-1315/451/1/012018

KOTHARKAR, R. et al. Evaluating urban heat island in the critical local climate zones of an Indian city. Landscape Urban Planning, v.169, p.92-104, 2018. doi:10.1016/j.landurbplan.2017.08.009

LEMUS-CANOVAS, M. et al. Estimating Barcelona's metropolitan daytime hot and cold poles using Landsat-8 Land Surface Temperature. Scie. Total Environ., v.699, p.134307, 2020. doi:10.1016/j.scitotenv.2019.134307

LIN, P. et al. Effects of urban planning indicators on urban heat island: a case study of pocket parks in high-rise high-density environment. Landscape Urban Planning, v.168, p.48-60, 2017. doi:10.1016/j.landurbplan.2017.09.024

LIU, S. et al. Estimating the surface air temperature by remote sensing in northwest china using an improved advection-energy balance for air temperature model. Adv. Meteorol., v.2016, p.4294219, 2016. doi:10.1155/2016/4294219

MARANDO, F. et al. Regulating Ecosystem Services and Green Infrastructure: assessment of Urban Heat Island effect mitigation in the municipality of Rome, Italy. Ecol. Modelling, v. 92, p.92102, 2019. doi:10.1016/j.ecolmodel.2018.11.011

NOVAIS, J. W. Z. et al. Variação Espaço-Sazonal Termohigrométrica do Parque Urbano Mãe Bonifácia pelo Método da Krigagem. Rev. Ensino, Educ. Ciênc. Hum., v.18, n.3, p.252, 2017. doi:10.17921/2447-8733.2017v18n3p252-257

NOVAIS, J. W. Z. et al. Índice de Temperatura e Umidade (ITU) Visando o Conforto Térmico para o Parque Mãe Bonifácia, Cuiabá - MT. Ensaios Ciênc., v.22, n.2, p.69-75, 2018. doi:10.17921/1415-6938.2018v22n2p69-75

PELTA, R. et al. Spatio-temporal behavior of brightness temperature in Tel-Aviv and its application to air temperature monitoring. Environ. Pollution, v.208, p.153-160, 2016. doi:10.1016/j.envpol.2015.09.007

PELTA, R. et al. Spatiotemporal estimation of air temperature patterns at the street level using high resolution satellite imagery. Scie. Total Environ., v.579, p.675-684, 2017. doi:10.1016/j. scitotenv.2016.11.042

SCHULTZ, M. et al. Performance of vegetation indices from Landsat time series in deforestation monitoring. Int. J. Appl. Earth Observation Geoinformation, v.52, p.318-327, 2016. doi:10.1016/j.jag.2016.06.020

SOUZA, A S. et al. Aplicações do sensoriamento remoto para estudos em arquitetura e urbanismo: subsídios para correlações entre dados remotos e medições de solo. In: SIMPÓSIO BRASILEIRO DE SENSORIAMENTO REMOTO - SBSR. SantosSP: INPE, 2017. p. 6514-6520.

USGS. 2017. Product Guide: Landsat Surface ReflectanceDerived Spectral Indices. Department of the Interior U.S. Geological Survey, version 3.6, p.16, 2017.

WANG, R. et al. Urban heat island and overheating characteristics in Sydney, Australia. An analysis of multiyear measurements. Sustainability (Switzerland), v.9, n.5, p.712, 2017. doi:10.3390/ su9050712

YANG, Chaobin et al. Mapping the influence of land use/land cover changes on the urban heat island effect-A case study of Changchun - China. Sustainability (Switzerland), v.9, n.2, p.312, 2017a. doi:10.3390/su9020312

YANG, A. et al. Numerical simulation of cooling effect of vegetation enhancement in a subtropical urban park. Applied Energy, v.192, p.178-200, 2017b. doi:10.1016/j. apenergy.2017.01.079

YU, Z. et al. How can urban green spaces be planned for climate adaptation in subtropical cities? Ecological Indicators, v. 82, p. 152-162, 2017. doi:10.1016/j.ecolind.2017.07.002

YU, Z. et al. Variations in land surface temperature and cooling efficiency of green space in rapid urbanization: The case of Fuzhou city, China. Urban Forestry and Urban Greening, v.29, p. 113-121, 2018. doi:10.1016/j.ufug.2017.11.008

ZHANG, R. et al. A remote sensing method for estimating surface air temperature and surface vapor pressure on a regional scale. Remote Sensing, v.7, n.5, p 6005-6025, 2015. doi:10.3390/ rs70506005

ZHANG, X. et al. Effects of land use/cover changes and urban forest configuration on urban heat islands in a loess hilly region: Case study based on yan'an city, China. Int. J. Environ. Res. Public Health, v.14, n.8, p.840, 2018. doi:10.3390/ijerph14080840

ZHOU, X. et al. CHEN, H. Impact of urbanization-related land use land cover changes and urban morphology changes on the urban heat island phenomenon. Scie. Total Environment, v.635, p. 1467-1476, 2018. doi:10.1016/j.scitotenv.2018.04.091 